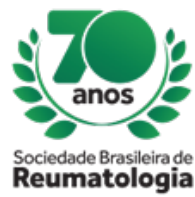

\title{
MYIASIS IN A PATIENT WITH GRANULOMATOSIS WITH POLYANGEITIS REMISSION IN USE OF RITUXIMABE
}

Raissa Velasques de Figueiredo (Universidade Federal de Ciências da Saúde de Porto Alegre, Porto Alegre, RS, Brasil), Maria Lúcia Lemos Lopes (Universidade Federal de Ciências da Saúde de Porto Alegre , Porto Alegre, RS, Brasil), Tatiana Freitas Tourinho (Universidade Federal de Ciências da Saúde de Porto Alegre, Porto Alegre, RS, Brasil), Maria Odete Esteves Hilário (Universidade Federal de Ciências da Saúde de Porto Alegre , Porto Alegre, RS, Brasil), Gilberto Scanagatta (Universidade Federal de Ciências da Saúde de Porto Alegre, Porto Alegre, RS, Brasil), Rafael Coradin (Universidade Federal de Ciências da Saúde de Porto Alegre, Porto Alegre, RS, Brasil), Luana Ribeiro Carlos (Universidade Federal de Ciências da Saúde de Porto Alegre, Porto Alegre, RS, Brasil), Thiago Willers (Universidade Federal de Ciências da Saúde de Porto Alegre, Porto Alegre, RS, Brasil), Eduardo Rosa de Oliveira (Universidade Federal de Ciências da Saúde de Porto Alegre, Porto Alegre, RS, Brasil), Alessandra Ferrari (Universidade Federal de Ciências da Saúde de Porto Alegre, Porto Alegre, RS, Brasil), Felipe Giovani Tawil Aubin (Universidade Federal de Ciências da Saúde de Porto Alegre , Porto Alegre, RS, Brasil), Gisa Moraes Muratt (Universidade Federal de Ciências da Saúde de Porto Alegre, Porto Alegre, RS, Brasil)

\section{BACKGROUND}

Polyangeitis granulomatosis is an associated ANCA vasculitis of small vessels that very often affects the lung, upper airways, and kidney. Its treatment consists of methylprednisolone pulse induction therapy, followed by maintenance therapy with cyclophosphamide. In refractory cases or in the failure of cyclophosphamide the use of rituximab to control the disease is indicated.

In this report, we present a case of a patient with granulomatosis with polyangeitis, with involvement of the lung, kidney and upper airways, using rituximab and presenting, 2 weeks before the next rituximab infusion, myiasis in the sinuses.

\section{CASE REPORT}

A 55-year-old female patient diagnosed with granulomatosis with polyangeitis since 2010 . At the time of diagnosis, the patient was treated with a pulse of methylprednisolone and maintained with oral cyclophosphamide and prednisone. It remained stable for about 2 years, when it started with worsening of sinusitis symptoms and hearing loss. It was then chosen to increase the dose of prednisone and that of cyclophosphamide. Patient remained with symptoms of the upper respiratory tract despite the adjustment of the immunosupression, being chosen then to change the cyclophosphamide by rituximab. In May of 2018 she presented intense headache, with exit of small larvae of the nasal cavity. Symptoms started 1 week before the new dose of rituximab. Patient was then referred to the emergency room and was evaluated by otorhinolaryngology that closed the diagnosis of myiasis of the sinuses after rigid nasal endoscopy showing visualization of larvae adjacent to the tail of the middle turbinate in the left nasal fossa. She also performed tomography of the breasts of the face that demonstrated larvae in the paranasal sinuses. Patient was treated with mechanical removal of the larvae plus ivermectin for 2 days and amoxicillin + clavulanate for 7 days, with improvement of the condition.

Patient evolved with improvement of clinical condition, currently using rituximab (4th dose) with partially controlled disease (persists with repetitive rhinosinusitis).

\section{CONCLUSION}

Granulomatosis with Polyangeitis is a vasculitis of small vessels that affects several systems but mainly affects the kidneys, lungs and airways. The maintenance of remission may be challenging and require more potent immunosuppression such as rituximab. Often, the use of such drugs brings the benefit of 
disease control over an increased risk of infections / infestations. In this case, we report a patient with granulomatosis with polyangeitis using rituximab who developed a myiasis infestation in the breasts of the face weeks before the next immunosuppressant infusion. 